J. Clin. Chem. Clin. Biochem.

Vol. 20, 1982, pp. 915-920

\title{
Methodische Untersuchungen verschiedener Filterpapierqualitäten im Rahmen der radioimmunologischen Thyrotropinbestimmung bei Neugeborenen
}

\author{
Von B. Höpfner \\ Aus dem Staatl. Medizinal-Untersuchungsamt (Leitung: Medizinaldirektor Dr. W. Werner) Dillenburg
}

(Eingegangen am 19. April/16. Juli 1982)

\begin{abstract}
Zusammenfassung: Im Rahmen des Neugeborenen-Screenings auf kongenitale Hypothyreose fungieren Filterpapierkarten als Probenträger für Kapillarblutproben und als Bezugsgröße für die zu ermittelnden Hormonkonzentrationen. Damit kommt deren Qualität besondere Bedeutung zu, der durch die Untersuchung einzelner Filterpapiersorten sowie verschiedener Markierungen für die Auftropffläche des Blutes Rechnung getragen wurde. Auch die Frage der Hormonverteilung innerhalb des getrockneten Blutstropfens sowie das Eluierverhalten des zu bestimmenden Hormons im Zusammenhang mit Empfindlichkeit und Ausbeute des Test-Kits wurden experimentell untersucht.
\end{abstract}

Study of the significance of different qualities of filter paper in the radioimmunological determination of thyrotropin in newborns

Summary: In the screening of newborns for congenital hypothyroidism, filter paper cards act as sample carriers for capillary blood specimens, and for reference quantities of the measured hormones. Therefore, the quality of the filter paper is especially important and must be taken into account. Different types of filter paper and different guide marks on the paper surface for spotting blood specimens were investigated.

The hormone distribution within the dried blood spot and the time necessary to extract the hormones were examined in relation to the sensitivity and yield of the test kit.

\section{Einführung}

Seit dem Jahre 1977 werden im Staatl. Medizinal-Untersuchungsamt Dillenburg Laboratoriumsuntersuchungen zur Diagnostik der kongenitalen Hypothyreose durchgeführt. Zunächst als Pilotstudie und ạb dem Jahre 1979 als flächendeckendes Vorsorgeprogramm für das Land Hessen werden nunmehr hier jährlich etwa $60000 \mathrm{Neu}$ geborrene auf dieses Krankheitsbild untersucht. Danach findet sich in unserem Klientel die angeborene Schilddrüsenunterfunktion bei einem von etwa 2800 Neugeborenen, und sie ist damit deutlich häufiger ạls die Phenylketonurie oder gar die Galaktosämie (1).

Bekanntlich führt die kongenitale Hypothyreose unbehandelt zu schweren irreparablen Entwicklungsstörungen des zentralen Nervensystems. Setzt aber die Behandlung in den ersten Lebenswochen ein, so treten keine Spätschäden auf (4). Deshalb wird bei allen Neugeborenen bereits am 5. Lebenstag eine Blutprobe zur Hormonbestimmung entnommen (zu diesem Zeitpunkt hat sich die bei gesunden Neugeborenen postpartal erhöhte Konzentration von Thyrotropin im Serum normalisiert und die Untersuchung kann gleichzeitig mit dem Test auf Phenylketonurie und Galaktosämie durchgeführt werden). Für die Analytik selbst wäre die Entnahme und das Weiterleiten einer Venenblutprobe zum Untersuchungslaboratorium zweifellos die optimale Lösung. Diese Forderung ist jedoch aus technischen und organisatorischen Gründen bei einem Massen-Screening - das alle Neugeborenen erfassen soll - nicht realisierbar. Aus praktischen Erwägungen hat man sich deshalb zwischenzeitlich der Verfahrensweise des Guthrie-Testes angeschlossen und tropft Kapillarblut aus der Ferse des Neugeborenen auf eine Filterkarte, die dann dem zentralen Untersuchungslabor zugeschickt wird. Dort eluiert man das Blut aus dem getrockneten Blutstropfen und bestimmt mit Hilfe eines spezifischen Radioimmunoassays die Konzentration des schilddrüsenstimulierenden Hormons (Thyrotropin, TSH).

Um zu verhüten, daß die relativ geringe $Z a h l$ von $\mathrm{zu}$ erwartenden pathologischen Befunden von einer großen Zahl falsch positiver oder falsch negativer Resultate übertroffen wird, müssen auch an die Präzision einer 
Screening-Methode hohe Anforderungen gestellt werden $(2,7)$. Da bei diesen Neugeborenen-Untersuchungen das Filterpapier sowohl als Probenträger als auch als Bezugsgröße fungiert, ist es notwendig, gerade der Qualität dieses Parameters besondere Aufmerksamkeit zu widmen (3). Deshalb wurde im Rahmen der vorliegenden Arbeit nicht nur der Einfluß verschiedener Filterpapierqualitäten auf die Ergebnisse der Thyrotropinbestimmungen geprüt, sondern auch die Frage der ThyrotropinVerteilung innerhalb des getrockneten Blutstropfens und das Eluierverhalten des zu bestimmenden Hormons näher untersucht.

\section{Material und Methodik}

Folgende Filterpapiersorten, die alle mit einem Flächengewicht von $180 \mathrm{~g} / \mathrm{m}^{2}$ angegeben werden, wurden geprüft:

\section{1) SS 2992 (Fa. Schleicher \& Schüll, D-3354 Dassel) \\ Das für das Hypothyreose-Screening z.Zt. meistverwendete $\mathrm{Pa}$ pier in Deutschland, das aber auch in anderen europäischen und außereuropäischen Ländern für den gleichen Zweck Ver- wendung findet.}

2) SS 903 (Fa. Schleicher \& Schüll, Keene, New Hampshire, USA)

Verwendung für das Hypothyreose-Screening u.a. in USA und Kanada.

3) MN 818 (Fa. Macherey-Nagel, D-5160 Düren)

Verwendung für das Hypothyreose-Screening in Deutschland und vereinzelt auch in europäischen und außereuropäischen Ländern.

4) MN 818 (4 Jahre) (Gleicher Hersteller und gleiche Qualität wie 3), jedoch mit einer Lagerzeit von 4 Jahren.

Zur Untersuchung selbst wurden Vollblutproben mit verschiedenen Thyrotropin-Konzentrationen angereichert und auf die zu analysierenden Testpapiere aufgetropft. Dabei wurde Thyrotropin-armes, heparinisiertes Blut mit dem WHO-Thyreotropinstandard MRC 68/38 versetzt und eine geometrische Verdünnungsreihe angelegt. Das Auftropfvolumen betrug jeweils $50 \mu$ l bei einem Thyrotropin-Konzentrationsbereich von 6,5 bis $100 \mathrm{mE} / \mathrm{l}$. Die aus den getrockneten Blutstropfen ausgestanzten Blättchen wiesén einen Kreisdurchmesser von $8 \mathrm{~mm}$, bei den "Lokalisationsversuchen" dagegen von $3 \mathrm{~mm}$ auf. In der Regel wurden 8fach-Bestimmungen mit 3fachen Wiederholungen ausgefuhrt. Zur Durchführung der eigentlichen Hormonanalysen fand ein handelsüblicher Radioimmunoassay Verwendung, der auf dem Prinzip der Doppelantikörpermethode basiert: Das ausgestanzte Blutblättchen wird mit Barbitalpuffer und Thyrotropin-Antiserum (Kaninchen) versetzt und über Nacht eluiert bzw. inkubiert. Danach folgt eine 5stündige Inkubation mit [ ${ }^{125}$ I] Thyrotropin, nach deren Ablauf das an den Antikörper gebundene Thyrotropin mit Hilfe von Polyethylenglykol und einem zweiten Antiserum (Anti-IgG, Ziege) gefällt wird. Nach der Zentrifugation des Ansatzes wird der Überstand abgesaugt und die Radioaktivität des Präzipitates ermittelt.

Hierzu diente ein 2-Kanal-Gammaprobenwechsler mit eingebautem Mikroprozessor zur Berechnung der Konzentrationswerte. Die Auswertung der Standardkurve erfolgte nach Punkt zu Punkt-Linearinterpolation, wobei $\mathrm{B} / \mathrm{B}_{0}$ gegen log-Konzentration aufgetragen wurde. Die Eingabe der Zähl- und Auswertegrößen sowie die Ausgabe der Konzentrationswerte und auch der Standardkurve erfolgte mit Hilfe eines elektronischen Blattschreibers mit Matrixdrucker (Teletype).

\section{Ergebnisse und Diskussion}

In Tabelle 1 werden die Ergebnisse der Untersuchungen über den Einfluß unterschiedlicher Filterpapiersorten auf die Resultate der Thyrotropin-Bestimmungen bei Neugeborenen wiedergegeben, wobei zunächst Filterpapiere ohne Markierung - also ohne Kennzèichnung der Blutauftropffläche - Verwendung fanden.

Tab. 1. Einfluß unterschiedlicher Filterpapiersorten (ohne Markierung) auf die Ergebnisse der Thyrotropin-Bestimmung.

\begin{tabular}{llccc} 
Papier- & \multicolumn{5}{c}{ Thyrotropinkonżentration $(\mathrm{mE} / \mathrm{l})$} \\
Sorte & 108 & 38 & 16 & 8 \\
\hline \multicolumn{5}{c}{ Relätive Wiederfindung } \\
SS 2992 & 1,00 & 1,00 & 1,00 & 1,00 \\
SS 903 & 1,16 & 1,26 & 1,12 & 1,00 \\
MN 818 & 1,07 & 1,13 & 1,06 & 1,00 \\
MN 818 & 1,06 & 1,13 & 1,06 & 1,00 \\
(4 Jahre) & & & & \\
\hline
\end{tabular}

Es. wird deutlich, daß die Filterpapier̃qualitäten SS 2992 und MN 818 in allen Konzentrationsbereichen ähnliche Thyrotropin-Werte zeigen und deshalb miteinander vergleichbar sind. Dies gilt nicht für die Papiersorte SS 903, sie weist - bezogen auf die Flächeneinheit - eine höhere Saugkapazität auf und liefert deshalb auch deutlich höhere Thyrotropin-Werte.

Zur Markierung der Blutauftropfflächen auf den Filter-karten werden national und international verschiedene Kennzeichnungen verwendet, die im wesentlichen die folgenden 4 Varianten beinhalten (Abb. 1):
$A=$ einseitig aufgedruckte Kreismarkierungen
$\mathrm{B}=$ beidseitig aufgedruckte K̇reismarkierungen
C $=$ beidseitig gepunktete Kreismarkierungen
$\mathrm{D}=$ gestanzte Kreismarkierungen

Um den Einfluß dieser verschiedenen Kreieskennzeich: nungen auf die Qualität der Thyrotropinbestimmungen zu ermitteln, wurden vergleichende Untersuchungsreihen auf Schleicher \& Schüll-Papier SS 2992 mit den o.a. Markierungen durchgeführt. Schon bei der makroskopischen Betrachtung der aufgetropften identischen Blutvolumina zeigt sich im Randbereich bei den Papieren B und D eine ungleichmäßige Verteilung, während die Papiere. A und $C$ homogenere Verteilungsverhältnisșe widerșpiegeln.

Dieser visuelle Eindruck wird bei der Auswertung der hierżu experimentell ermittelten Untersuchungșbefunde gestützt (Tab. 2): Einseitig bedruckte und beidseitig gepunktete Papiere liefern weitgehend idèntische und damit vergleichbare Ergebnisse. Hiervon stark abweichende Resultate finden sich dagegen vor allem bei dem beidseitig kreisbedrucktem Papier. Auch die Werte für die gestanzten Papiere zeigen gegenüber den beiden erstgenannten Filterpapieren abweichende, ;Tendenzen. 

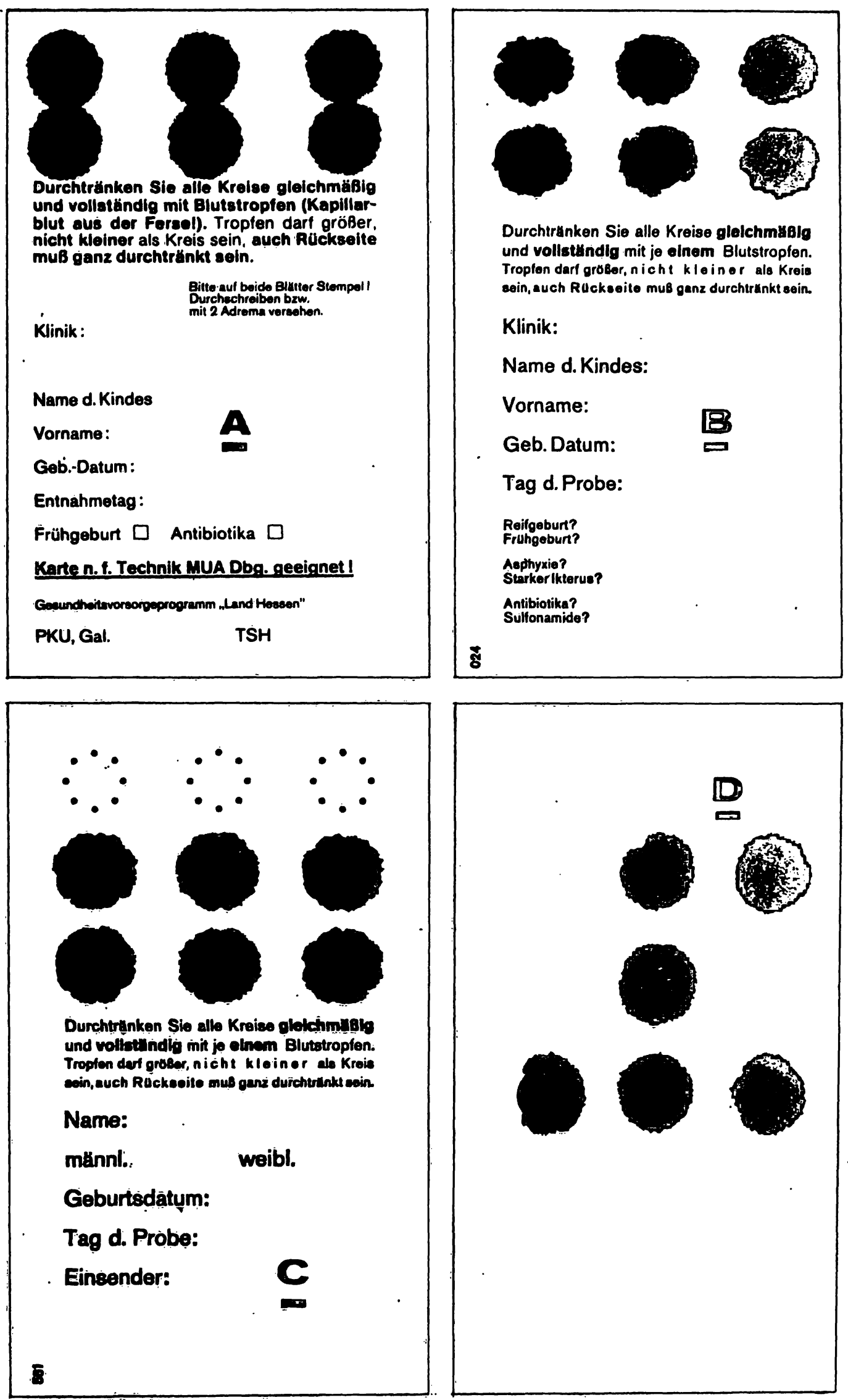

Abb. 1. Kennzeichnungen zur Markierung der Blutauftropfflächen auf den Filterkarten.

$A=$ einseitig aufgedruckte, $B=$ beidseitig aufgedruckte, $C=$ beid'seitig gepunktete, $D=$ gestanzte Kreismarkierung. 
Tab. 2. Einfluß unterschiedlicher Kreiskennzeichnungen auf die Ergebnisse der Thyrotropin-Bestimmung mit Papier SS 2992.

\begin{tabular}{lllll}
\hline \multirow{2}{*}{$\begin{array}{l}\text { Papier- } \\
\text { Markierung }\end{array}$} & \multicolumn{5}{l}{ Thyrotropinkonzentration (mE/1) } \\
& 98 & 35 & 15 & 8 \\
\hline & \multicolumn{5}{l}{ Relative } & \multicolumn{3}{c}{ Wiederfindung } \\
& 1,00 & 1,00 & 1,00 & 1,00 \\
$\begin{array}{l}\text { SS } 2992 \\
\text { einseitig bedruckt }\end{array}$ & 1,66 & 1,40 & 1,47 & 1,00 \\
$\begin{array}{l}\text { SS 2992 } \\
\text { beidseitig bedruckt }\end{array}$ & 1,05 & 1,09 & 0,93 & 1,00 \\
$\begin{array}{l}\text { SS 2992 } \\
\text { beidseitig gepunktet }\end{array}$ & 0,83 & 0,91 & 1,00 & 0,88 \\
$\begin{array}{l}\text { SS 2992 } \\
\text { gestanzt }\end{array}$ & 1,10 & 1,09 & 1,07 & 1,00 \\
$\begin{array}{l}\text { SS 2992 } \\
\text { unbedruckt }\end{array}$ & & & & \\
\hline
\end{tabular}

Diese gravierenden Unterschiede zwischen den einzelnen Kennzeichnungsarten wurden zunächst nicht erwartet. Eine Antwortiauf dieses überraschende Ergebnis sollten aber Verteilungsstudien des Thyrotropins innerhalb des getrockneten Blutstropfens liefern. Hierzu wurden zunächst auf den 4 verschiedenen unbedruckten Papiersorten jeweils $50 \mu \mathrm{l}$ Vollblut mit einer konstanten Thyrotropin-Konzentration aufgetropft (Tab. 3). Die gewählten Analysenpunkte sind aus dem Kreisdiagramm ersichtlich und symbolisieren die Kreismitte (= A), den Randbezirk des Blutstropfens $(=\mathrm{C})$ und das Areal zwischen den Punkten A und C (=B).

Tab. 3. Einfluß verschiedener Filterpapiersorten auf die Thyrotropin-Verteilung innerhalb der Auftropffläche (ohne Markierung).

\begin{tabular}{lllll} 
Papier- & \multicolumn{5}{l}{ Entnahme-Areale } \\
Sorte & A & B & C \\
\hline \multicolumn{5}{l}{ Relativer Anteil } \\
SS 2992 & 1,00 & 1,03 & 1,23 & (C) \\
SS 903 & 1,10 & 1,12 & 1,32 & (B)(B) \\
MN 818 & 1,03 & 1,09 & 1,27 & (C) \\
MN 818 & 1,06 & 1,11 & 1,18 & \\
(4 Jahre) & & & &
\end{tabular}

Die Auswertung der Analysenergebnisse ergibt, daß sich bei allen untersuchten Papieren in der Kreismitte die niedrigsten und im Randbezirk des Blutstropfens die höchsten Thyrotropin-Konzentrationen finden. Es tritt also ein deutlicher Chromatographie-Effekt auf, der wie auch Abbildung 2 zeigt - durch eine beachtenswerte Thyrotropin-Akkumulation im Randbereich des Blutstropfens gekennzeichnet ist. Beim Vergleich der 4 verschiedenen Papiere untereinander zeigen die Sorten SS 2992 und MN 818 weitgehend identische Ergebnisse, während die amerikanische Papiersorte SS 903 und das 4 Jahre gelagerte Papier MN 818 durch etwas abweichende Resultate auffallen.

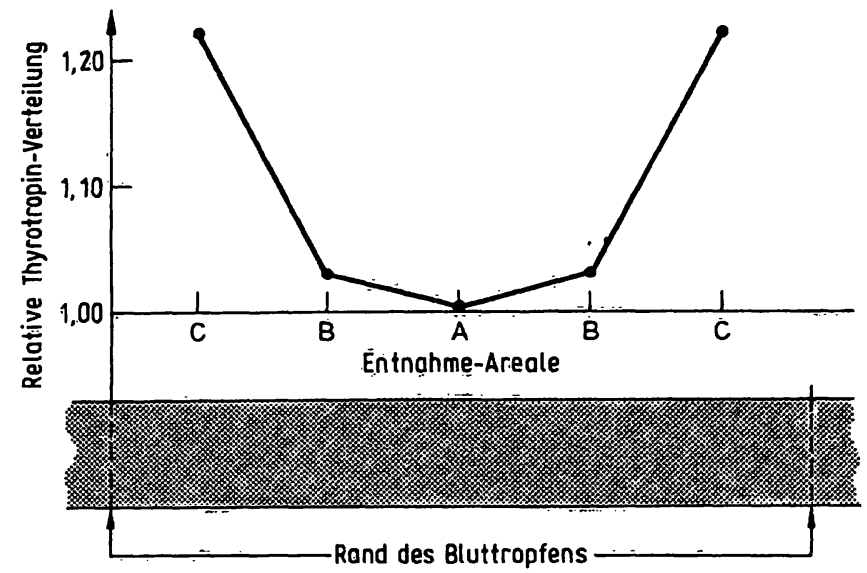

Abb. 2. Thyrotropin-Verteilung auf unbedrucktem Filterpapier.

Im zweiten Teil dieser Lokalisationsstudien wurden nun die verschiedenen Kreismarkierungen in die Untersuchungen einbezogen, wobei - wie schon zuvor - dás Papier SS 2992 als einheitliches Trägermaterial für die jeweiligen Kennzeichnungsarten diente. Auf Tabelle 4 findet sich neben den Ergebnissen auch wieder ein Kreisdiagramm mit den Punkten, die analytisch bei dieser Untersuchung berücksichtigt wurden; $A=$ Kreismitte, $B=$ Randbereich vor der Kreismarkierung und $\mathrm{C}=$ Randbezirk des Blutstropfens einsșchließlich einès Areals der Kreismarkierung selbșt. Màn sieht, daß außer den beidseits bedruckten Papieren die übrigen Kennzeichnungsvarianten kein wirkliches. Hindernis für die Diffu-

Tab. 4. Einfluß unterschiedlicher Kreiskennżeichnungèn der Filterpapierkarten auf die Thyrotropin-Verteilung innerhalb der Auftropffiäche mit Papièr SS 2992.

\begin{tabular}{|c|c|c|c|c|}
\hline \multirow{2}{*}{$\begin{array}{l}\text { Papier- } \\
\text { Markierung }\end{array}$} & \multicolumn{3}{|c|}{ Entnahme-Areale } & \\
\hline & $\mathbf{A}$ & B & $\mathrm{C}$ & \\
\hline & \multicolumn{3}{|c|}{ Relativer Anteil } & \\
\hline $\begin{array}{l}\text { SS } 2992 \\
\text { einseitig } \\
\text { bedruckt. }\end{array}$ & 1,00 & 1,01 & 1,10 & \\
\hline $\begin{array}{l}\text { SS } 2992 \\
\text { beidseitig } \\
\text { bedruckt }\end{array}$ & 1,00 & 1,23 & 1,07 & \\
\hline $\begin{array}{l}\text { SS } 2992 \\
\text { beidseitig } \\
\text { gepunktet }\end{array}$ & 1,00 & 1,03 & 1,16 & (B) \\
\hline $\begin{array}{l}\text { SS } 2992 \\
\text { gestanzt }\end{array}$ & 1,00 & 1,01 & $1,0 \overline{7}$ & \\
\hline $\begin{array}{l}\text { SS } 2992 \\
\text { unbedruckt }\end{array}$ & 1,00 & 1,03 & 1,23 & \\
\hline
\end{tabular}

sion des Thyrotröpins in den Randbezirk des Blutstropfens darstellen. Im weiteren Sinne lașsen sich diese Resultate sogar mit denen der nichtğekennzeichneten Filterpapiere vergleichen
: 
Völlig andere Verhältnisse ergeben sich jedoch bei beidseits bedruckten Papieren: In diesem Falle bildet die Kreismarkierung eine deutliche Barriere für das Thyrotropin. Es kommt - wie auf Abbildung 3 graphisch dargestellt - zu einer Anhäufung des Hormons vor der durch den Aufdruck gestauchten Markierungszone. Da dieses Ergebnis sich doch sehr stark von den übrigen Untersuchungsresultaten unterscheidet, sollten zukünftig Filterpapiere mit beidseits aufgedruckten (und geschlossenen) Kreismarkierungen nicht mehr für das Hypothyreose-Screening herangezogen werden.

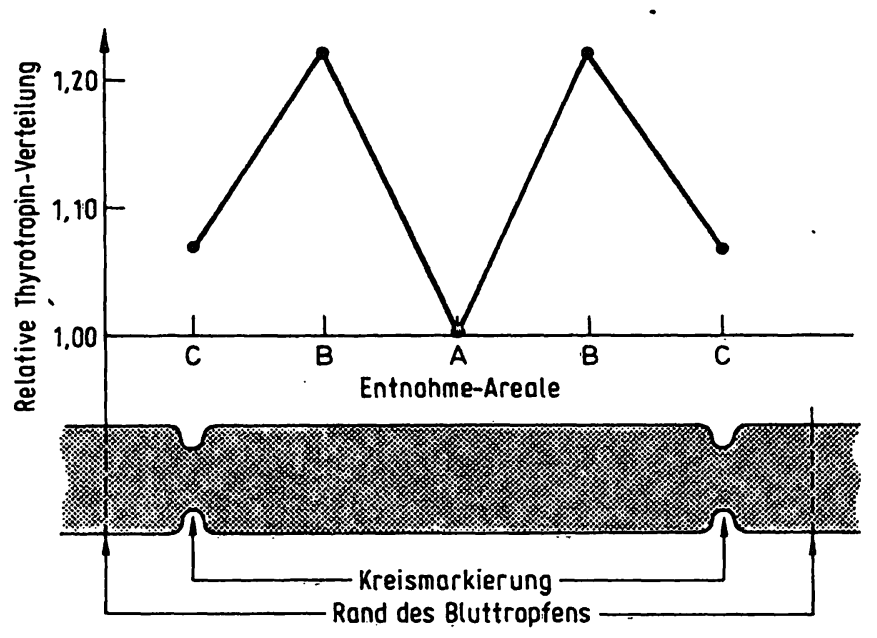

Abb. 3. Thyrotropin-Verteilung auf beidseitig bedrucktem Filterpapier.

Schließlich wurde noch im Rahmen der ThyrotropinAnalytik die Frage des Einflusses der Extraktionsdauer näher untersucht. Signifikante Unterschiede zwischen den einzelnen Papiersorten lassen sich jedoch nicht erkennen (Abb. 4). Beachtenswert erscheint aber, daß bereits 4 Stunden nach dem Probenansatz - bezogen auf das 18-h-Ergebnis - ein Anteil von über 0,95 des Thyrotropins bei allen Filterpapieren eluiert werden konnte. Eine merkliche Verkürzung der Gesamtanalysendauer ist aufgrund dieses Ergebnisses durchaus möglich, so daß selbst bei größerem Probenanfall alle Befunde bereits am Tag nạch dem Analysenansatz rechtzeitig vorliegen. Im Hinblick auf die absolute Thyrotropin-Ausbeute und die Empfindlichkeit der Doppelantikörpermethode neigt der Verfasser jedoch dazu, die ,überNacht"-Inkubation zu wählen, da hierdurch die AntigenAntikörperbindung günstig beeinflußt werden kann und auch arbeitstechnische Vorteile resultieren $(5,6)$.

Die bei dem Filterpapier MN 818 (alt) beobachtete geringe Reduzierung des 18 -h-Ergebnisses gegenüber dem 4-Stunden-Wert könnte durch Depolymerisationsprozesse - die bei Filterpapieren mit einer längeren Lagerzeit verstärkt auftreten - verursacht worden sein.

Aufgrund der vorliegenden Untersuchungsergebnisse scheint es nicht nur sinnvoll, sondern auch notwendig,
Filterpapiere für Vorsorgeuntersuchungen zu standardisieren. Dazu sollte der Deutsche Normenausschuß eine DIN-Norm erarbeiten, in der neben dem Grammgewicht auch die Saugkapazität bzw. das Saugvolumen bindend festgelegt wird. Diese, im Sinne der Qualität gerade des Hypothyreose-Screenings wünschenswerte Forderung ist nach den hier vorliegenden Erkenntnissen auch von seiten der Filterpapierhersteller technisch ohne größeren finanziellen Aufwand durchaus realisierbar.

Ein diesbezüglicher Antrag an den Deutschen Normenausschuß wurde deshalb zwischenzeitlich vom Verfasser eingereicht.

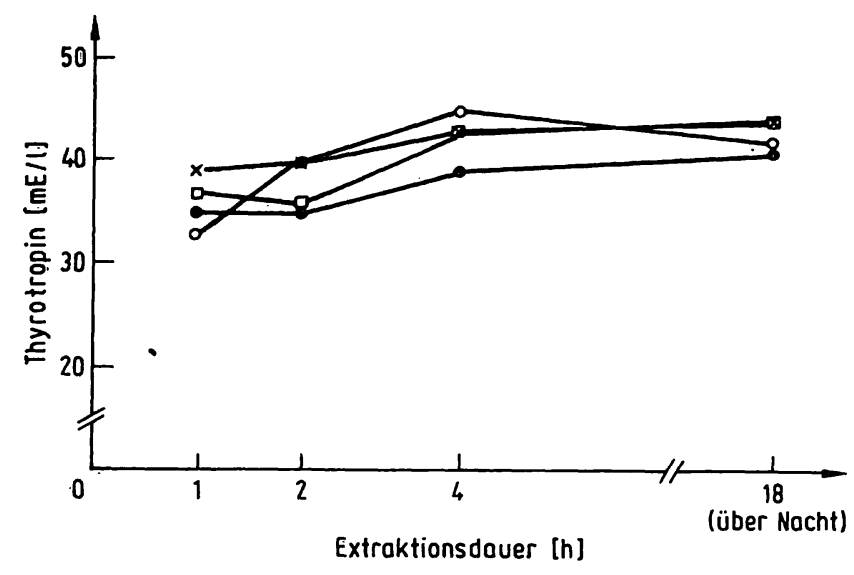

Abb. 4. Einfluß der Extraktionsdauer verschiedener Filterpapiersorten auf die Ergebnisse der Thyrotropin-Bestimmung.

\section{Fazit}

1. Für die Thyrotropin-Bestimmung bei Neugeborenen sollten nur Filterpapiere einheitlicher Qualität Verwendung finden (z.B. SS 2992 oder MN 818).

2. Zum Kennzeichnen der Blutauftropfflächen können folgende Markierungsmöglichkeiten empfohlen werden:

a) Kreis einseitig bedruckt

b) Kreis beidseitig gepunktet

3. Aufgrund der unterschiedlichen Thyrotropin-Verteilung innerhalb des getrockneten Blutstropfens lassen sich repräsentative Analysenergebnisse nur mit Hilfe großer Ausstanzflächen (z.B. 8 mm-Blättchen) erzielen.

4. Die geprüften Filterpapiersorten reagieren hinsichtlich der Extrahierbarkeit des Thyrotropins sehr ähnlich. Um die Hormonausbeute und die Empfindlichkeit des TestKits zu erhöhen, wird grundsätzlich eine ,über-Nacht"Inkubation empfohlen.

5. Zur Steigerung der Qualität des HypothyreoseScreening sollte der Deutsche Normenausschuß eine DIN-Norm erarbeiten, in der sowohl das Flächengewicht als auch die Saugkapazität bzw. das Saugvolumen bindend vorgeschrieben werden. 


\section{Literatur}

1. Delange, F., Beckers, C., Höfer, R., König, M. P., Monaco, F. \& Varrone, S. (1980) "Progress report on neonatal screening for congenital hypothyroidism in Europe", New York.

2. Hannon, W. H., Adam, B. W. \& Bayse, D. D. (1980) "The international status of thyroid-stimulating hormone asșays for neonatal hypothyroid screening", Atlanta, Georgia.

3. Holmlund, E. (1981) "Development work with PhadebasDry-Spot-Test using different qualities of standardpaper". Pharmacia-Identification, Freiburg.

4. Illig, R. \& de Vera Roda, C. R. (1976) Schweiz. Med. Wochenschr. 106, 1676-1681.
5. Sadler, W. A. \& Lynskey, C. P. (1979) Clinical Chemistry $25,933-938$.

6. Thiel, D. van, Marschner, I., Wood, W. G., Habermann, J. \& Scriba, P. C. (1980) J. Clin. Chem. Clin. Biochem. 18, 807816.

7. U.S. Department of Health, Education, arid Welfare (1979) "Proceedings of a conference on a national model for standardization of neonatal hypothyroid screening programs", Atlanta, Georgia.

Dr. Bernhard Höpfner

Staatl. Medizinal=Untërsuchụngșämt Wolframstraße 33

D-6340 Dillenburg 